\title{
Genetic screen identifies suppressor of morphogenesis in genitalia-1 (SMG-1) as a modulator of sorafenib resistance in hepatocellular carcinoma cell lines
}

\author{
SOON WOO NAM ${ }^{1}$, KI CHEOL PARK ${ }^{2}$, KEUM JIN YANG ${ }^{2}$, BYOUNGCHUN LEE ${ }^{3}$ and SUNG-WOO KIM ${ }^{3}$ \\ ${ }^{1}$ Hepatobiliary Unit, Division of Gastroenterology, Department of Internal Medicine, Incheon St. Mary's Hospital, \\ Medical School of the Catholic University of Korea, Incheon; ${ }^{2}$ Clinical Medicine Research Institute, \\ Daejeon St. Mary's Hospital, Daejeon, Republic of Korea; ${ }^{3}$ Southern Alberta Cancer Research Institute, \\ Departments of Biochemistry and Molecular Biology, University of Calgary, AB, Canada
}

Received August 5, 2013; Accepted October 7, 2013

DOI: $10.3892 /$ ijo.2014.2540

\begin{abstract}
Hepatocellular carcinoma (HCC) is an aggressive malignancy with a poor prognosis and a very complex dysregulated molecular etiology. Furthermore, conventional therapy thus far has had only limited success. A recently developed oral multikinase inhibitor, sorafenib, has been used to improve survival in HCC patients, however, follow-up studies have revealed a high rate of cancer recurrence. Therefore, identification of genes involved in sorafenib resistance is urgently required. RNA interference (RNAi) is a powerful tool for performing loss-of-function genetic screens and can facilitate the identification of components of the cellular signaling pathway. This study describes the results of an unbiased genomic screening using RNAi in an HCC cell line to elucidate genes related to sorafenib non-responsiveness or resistance. A genome-wide in vitro RNA interference screen revealed the role of suppressor of morphogenesis in genitalia-1 (SMG-1) as a determinant of sorafenib resistance. The inhibition of SMG-1 reduced sorafenib sensitivity in the studied HCC cell lines. An immunohistochemical comparison of cancerous and non-cancerous regions showed strong staining in the non-neoplastic hepatocyte regions of HCC. SMG-1 may warrant investigation as an agent to reverse sorafenib resistance.
\end{abstract}

\section{Introduction}

Hepatocellular carcinoma (HCC) is one of the most common malignant neoplasms in humans and is more prevalent among Asian and African populations (1,2). Molecular analyses have

Correspondence to: Professor Sung-Woo Kim, Southern Alberta Cancer Research Institute, Departments of Biochemistry and Molecular Biology, University of Calgary, 3330 Hospital Drive, Calgary, AB T2N 4N1, Canada

E-mail: swkim@ucalgary.ca

Key words: hepatocellular carcinoma, RNA interference, sorafenib, suppressor of morphogenesis in genitalia-1 shown that HCC pathogenesis is a multifactorial and multistep process reflecting alterations derived from epigenetic instability, chromosomal instability and deregulated transcription (3). Risk factors for HCC development include viral infections such as hepatitis B or C, exposure to aflatoxin B, chronic alcohol ingestion and cirrhosis from variable causes (4-6). This advanced or relapsing disease has a poor prognosis due to underlying liver disease and lack of effective systemic treatment. Moreover, conventional chemotherapy has not been demonstrated to prolong survival of patients with advanced HCC $(7,8)$. However, recent advances in our knowledge of HCC biology have led to the development of efficient agents to treat HCC (9).

Sorafenib (Nexavar, BAY 43-9006), an orally active multikinase inhibitor, has been used to treat several malignant neoplasms such as HCC and renal cell carcinoma (10). Although monotherapy with sorafenib has been shown to significantly prolong overall survival and delay time to progression in patients with advanced HCC, the recent reports have disappointingly been unable to substantiate a reduction of tumor burden in patients with advanced HCC (11-13). Sorafenib is currently the only accepted systemic chemotherapeutic used in the treatment of HCC, and as such, there is an urgent need to elucidate the genes that diminish its effectiveness.

RNA interference (RNAi) is a powerful new tool for performing loss-of-function genetic screens in lower organisms and can greatly facilitate the identification of components of cellular signaling pathways $(14,15)$. By suppressing gene expression and therefore, protein function to a certain extent, RNAi models the pharmacological inhibition of a target protein and can be an effective tool for proof-of-principle experiments to identify and validate cancer drug targets (16).

We performed an unbiased genetic screen on Hep3B human HCC cancer cell lines using a short hairpin RNA (shRNA) library that identified SMG-1 as a modulator of sorafenib sensitivity in vitro and executed the validation procedures to confirm whether the resultant sequenced target gene can be a significant molecular target for resistance to sorafenib treatment of HCC. 


\section{Materials and methods}

Cell culture. Hep3B, HepG2 and Huh7 cells (American Tissue Culture Collection) were seeded at a density of $1 \times 10^{6} \mathrm{~cm}^{2}$ and routinely cultured in Dulbecco's modified Eagle's medium (DMEM, Invitrogen Life Technology Inc.) supplemented with $10 \%$ fetal bovine serum plus penicillin-streptomycin $(10 \mathrm{IU} / \mathrm{ml})$ and fungizone $(2.5 \mu \mathrm{g} / \mathrm{ml})$. Cultures were maintained at $37^{\circ} \mathrm{C}$ in a humidified $5 \% \mathrm{CO}_{2}$ atmosphere.

Establishment of the retroviral vector $(p R S)$ - and $p R S$ $s h R N A-H e p 3 B$ cell line. For viral production, $2 \mu \mathrm{g}$ of retroviral vector $\mathrm{pRS}$ and $\mathrm{pRS}$ shRNA library were transfected into 293 Ampho cell lines $\left(1 \times 10^{6}\right)$. After 4 days of transfection their supernatants were infected into Hep3B cell lines and selected with $1.0 \mu \mathrm{g} / \mathrm{ml}$ puromycin as previously described (17).

siRNA transfection. Three Stealth ${ }^{\mathrm{TM}}$ RNAi duplexes were synthesized commercially by Invitrogen Life Technologies Inc. with the help of tools available online (http://www.invitrogen.com). Stealth RNAi lines (HSS118096, HSS118097, HSS11808) were designed to target different coding regions of the human SMG-1 mRNA sequence (NCBI Reference Sequence: NM_015092.3). Hep3B, HepG2 and Huh7 cells were seeded at $4 \times 10^{5}$ cells per well in a $60-\mathrm{mm}$ dish in DMEM (Invitrogen Life Technology Inc.) containing $10 \%$ fetal bovine serum (Invitrogen Life Technology Inc.). On the following day, cells were transfected with the three Stealth RNAi duplexes as directed by the manufacturer's protocol for the Hiperfect transfection reagent (Qiagen). The final concentration of Stealth RNAi was $50 \mathrm{nM}$. A control also contained siRNA Negative Control Med GC (Invitrogen Life Technology Inc.).

Reverse transcription and real-time $q P C R$. Total RNA was extracted from Hep3B using NucleoSpin RNA II (Macherey-Nagel, Düren, Germany). The $1 \mu \mathrm{g}$ of total RNA was reverse-transcribed using Reverse Transcriptase Premix (Elpis Biotech, Daejeon, Korea) according to the manufacturer's instructions. The probe for TaqMan PCR was purchased from Invitrogen and real-time PCR was performed using the 7500 Fast Real-Time PCR System (Applied Biosystems, Foster City, CA). Glyceraldehyde-3-phosphate dehydrogenase was used as an internal standard and used to normalize the $\mathrm{Ct}$ values.

Western blot analysis. Cells were washed with PBS and harvested in lysis buffer.Proteins were separated using NuPAGE Novex 3-8\% Tri-Acetate (Invitrogen Life Technology Inc.). All primary antibodies were diluted in 3\% BSA in Tris-buffered saline with $0.1 \%$ Tween (TBS-T) at the following dilutions: anti- $\beta$-actin (1:1,000, Cell Signaling Technology), anti-SMG-1 (1:500, Cell Signaling Technology) and secondary HRP conjugated antibodies (Cell Signaling Technology). HRP was detected using an Immunobilon Western Chemiluminescent HRP substrate kit (Millipore). Images were scanned using a Bio-Rad ChemiDoc XRS System.

Cell proliferation assay. The cells were treated with 0-10 $\mu \mathrm{mol}$ of sorafenib. Following a 48-h incubation period, cellular proliferation was assessed using the EZ-CyTox cell viability assay kit (Daeillab, Korea). EZ-CyTox solution $(10 \mu \mathrm{l})$ was added to each cell cultured 96-well, and the mixtures were incubated for $2 \mathrm{~h}$ at $37^{\circ} \mathrm{C}$. Absorbance was then measured using an ELISA reader at $450 \mathrm{~nm}$ (microplate reader, Bio-Rad). Cellular proliferation was expressed as a percentage of vehicle-treated cells, which were defined as $100 \%$ viable.

Immunohistochemical staining. To investigate the expression of SMG-1 in 20 human hepatocellular carcinoma tissue samples, we performed immunohistochemical staining by using antiSMG-1 (1:200, Cell Signaling Technology) to tissues that had been surgically resected or biopsied after informed consents. Enrolled patients had no previous sorafenib treatment. We designated the expression strengths as 1 (weakest staining), 2, 3 and 4 (strongest staining) after meticulous investigation of tissue slides and then compared the expression strengths of the cancer region with non-cancerous regions of resected tissue samples.

Statistical analysis. Single group comparisons were done with two-tailed Student's t-tests and one-way Anova.

\section{Results}

Establishment of Hep3B cell lines containing the shRNA screening library. A schematic diagram shows the screening strategy employed to identify sorafenib-resistant genes from the stage of puromycin selection to sequencing of target genes (Fig. 1A). Hep3B cells known to have high sorafenib sensitivity (18) were infected with the empty retrovirus-based pRS vector or the pRS-shRNA library that can suppress 8,000 human genes (19) and selected with puromycin $(1.0 \mu \mathrm{g} / \mathrm{ml})$ for 2 weeks. During puromycin selection, mock cells were all dead, whereas more than $80 \%$ of the cells containing pRS vector and pRS-shRNA library survived (Fig. 1B).

shRNA screen identifies genes involved in sorafenib resistance. To determine the optimal dose for shRNA library selection, Hep3B mock or Hep3B-pRS cells were treated with sorafenib (0 to $10 \mu \mathrm{M})$ for 4 days. The effect of sorafenib on cell viability was measured by the WST-1 assay. Sorafenib inhibited cell viability dose-dependently (Fig. 2A). The maximal effect of sorafenib on the viability of Hep3B or Hep3B-pRS cells was shown at the concentration of $10 \mu \mathrm{M}$. This dose was also shown to be the highest concentration producing acquired resistance to sorafenib in HCC (20). Based on these findings, the pRS-shRNA library or pRS vector-infected Hep3B cells were cultured in the presence of sorafenib $(10 \mu \mathrm{M})$ for 1 to 4 days. An increase in viability of shRNA library-infected cells was observed with 3- and 4-day sorafenib treatments after the third screening using the WST assay (Fig. 2B). Three successive rounds of screening led to the identification of shRNA for SMG-1 (shSMG-1) which was present in sorafenib-resistant third screened shRNA libraryinfected cells after each round (Fig. 2C) and also identified five other genes for investigation.

Suppression of SMG-1 leads to sorafenib resistance in HCC cell lines. To confirm the effect of suppressed SMG-1 

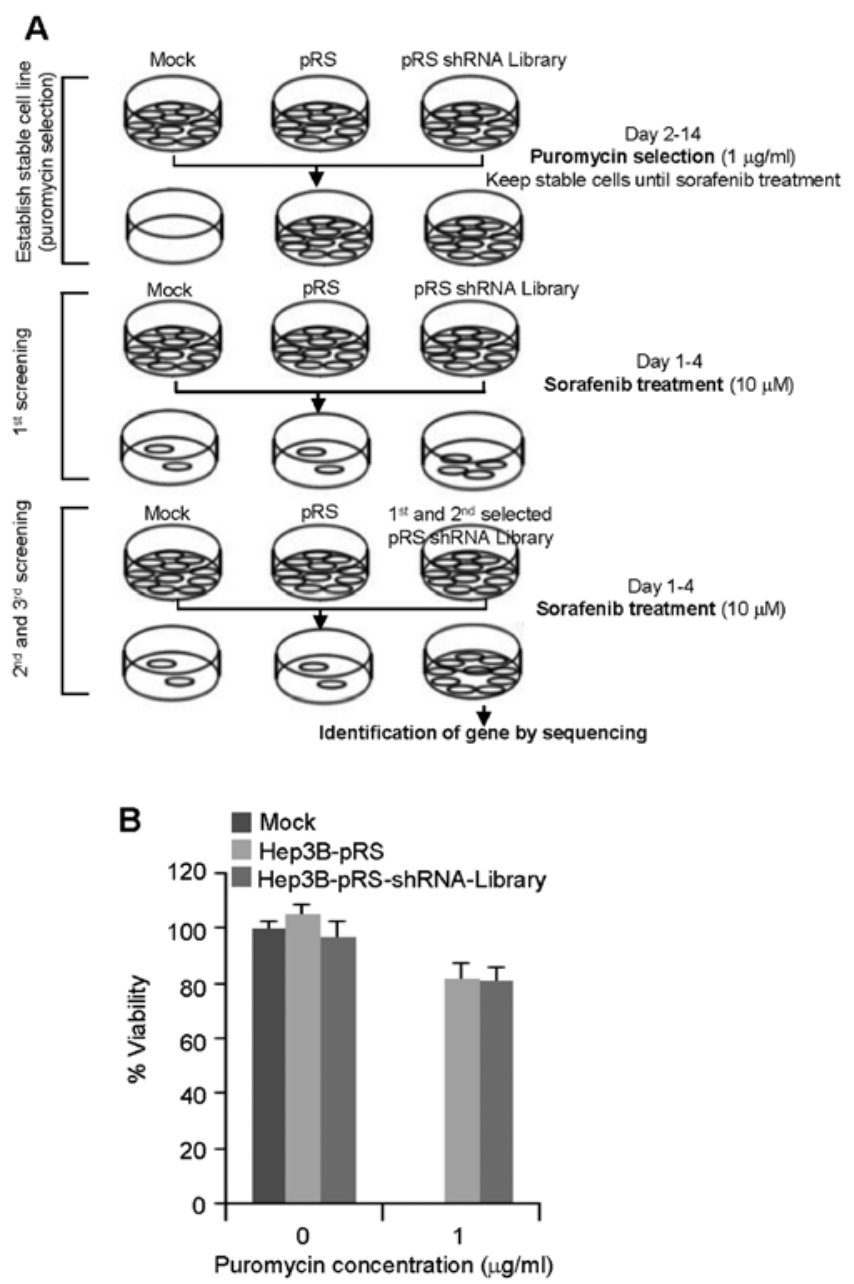

Figure 1. Schematic strategy of shRNA library screening. (A) A schematic diagram shows the establishment of Hep3B cell lines containing shRNA screening library and the strategy from puromycin selection to target gene identification. (B) Viability of mock, pRS, pRS-shRNA infected cells after puromycin selection.

expression on sorafenib resistance, three specific RNAi oligonucleotides of SMG-1 were transfected into the Hep3B cell line. Among them, the cells transfected with si-SMG-1 \#6 effectively suppressed the SMG-1 mRNA transcripts (Fig. 3A) and proteins (Fig. 3B) at the concentrationof $50 \mathrm{nM}$. After the transfection with si-SMG-1 \#6, the cells were treated with sorafenib at the indicated doses for 24 and $48 \mathrm{~h}$ and assayed for cell viability. Significantly higher viability at day $1(\mathrm{p}<0.05)$ and day $2(\mathrm{p}<0.01)$ was shown in the si-SMG-1-transfected cells compared to the control at the 5 and $10 \mu \mathrm{M}$ concentrations of sorafenib treatment in Hep3B cells.

To investigate these results in other HCC cell lines, HepG2 and Huh7 cell lines were transfected with either si-SMG-1 \#6 or si-scramble. SMG-1 RNAi suppressed the SMG-1 mRNA level to 80 and $70 \%$ at the $100 \mu \mathrm{M}$ concentration, as seen in the HepG2 and Huh7 cell lines, respectively (Fig. 4A). Similarly, suppression of the SMG-1 resulted in significantly higher cell viability at the $10 \mu \mathrm{M}(\mathrm{p}<0.05)$ and $20 \mu \mathrm{M}(\mathrm{p}<0.01)$ concentrations of sorafenib in both cell lines (Fig. 4B). Our data suggest that the suppression of SMG-1 may increase sorafenib resistance in HCC.
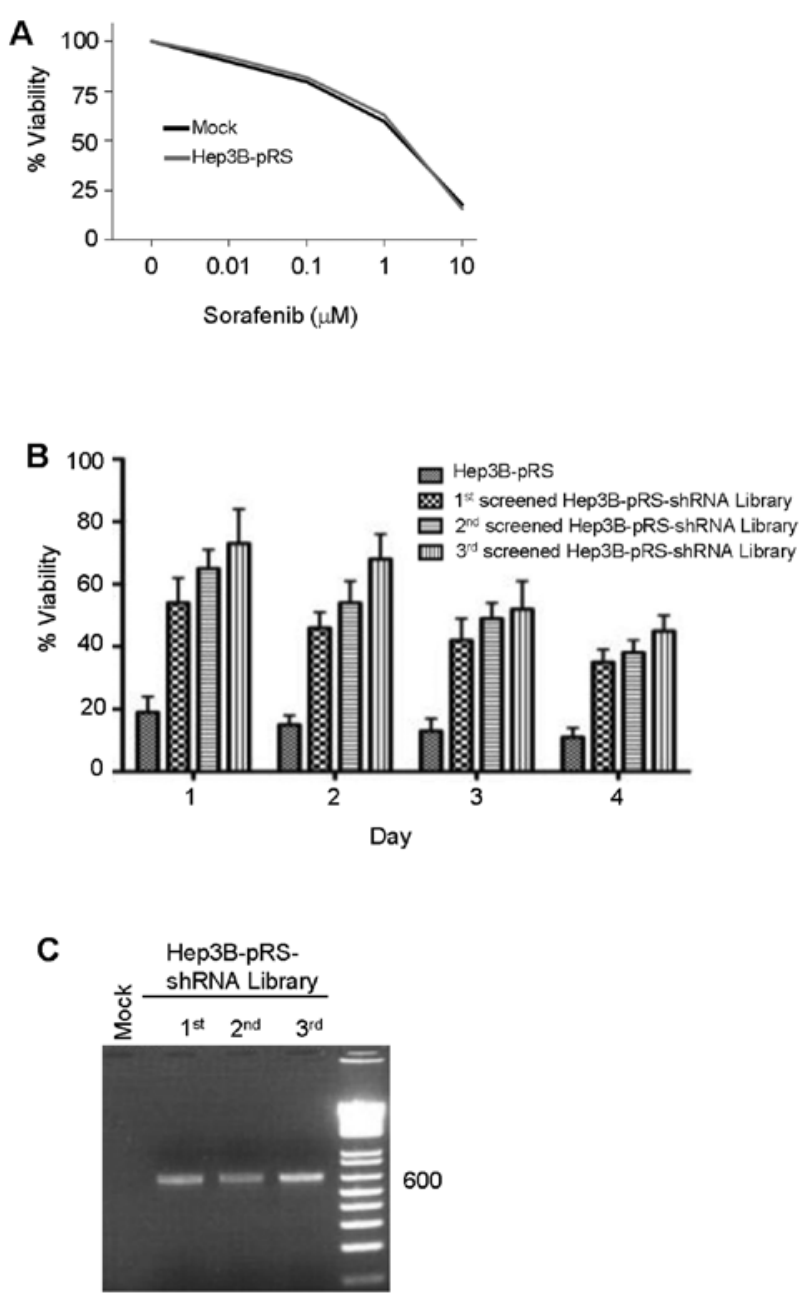

Figure 2. Genetic screening identifies knockdown of SMG-1 in development of sorafenib resistance. (A) Hep3B cells were treated with sorafenib (4 days) and viability was assessed with WST1 assays. (B) After each round of screening, viability of survived cells after sorafenib treatment $(10 \mu \mathrm{M})$ was assessed using WST1. (C) Hep3B cells were subjected to genetic screening for sorafenib resistance with pRS-shRNA library, genomic DNA was isolated, shRNA insert was amplified by PCR and products were resolved on agarose gels.

Immunohistochemical staining results show reduced SMG-1 expression in cancerous regions. Immunohistochemical staining of SMG-1 in cancerous and non-cancerous regions of surgically resected tissues (Fig. 5A and B) showed reduced expression in cancerous regions compared to non-cancerous regions and controls. On the other hand, there was stronger cytoplasmic granular staining in non-cancerous regions $(\mathrm{p}<0.01)$.

\section{Discussion}

We applied the shRNA library-based genetic approach to identify genes related to the sorafenib resistance of human HCC cell lines. As expected, an increase in the viability of shRNA library-infected cells was observed with 3- and 4-day sorafenib treatments after the third screening compared to controls. The value of this approach is that it allows the least biased identification of new and unanticipated tumor suppressors. In this regard, our study provides significant evidence for the identification of the novel tumor suppressor SMG-1. Successive validation 

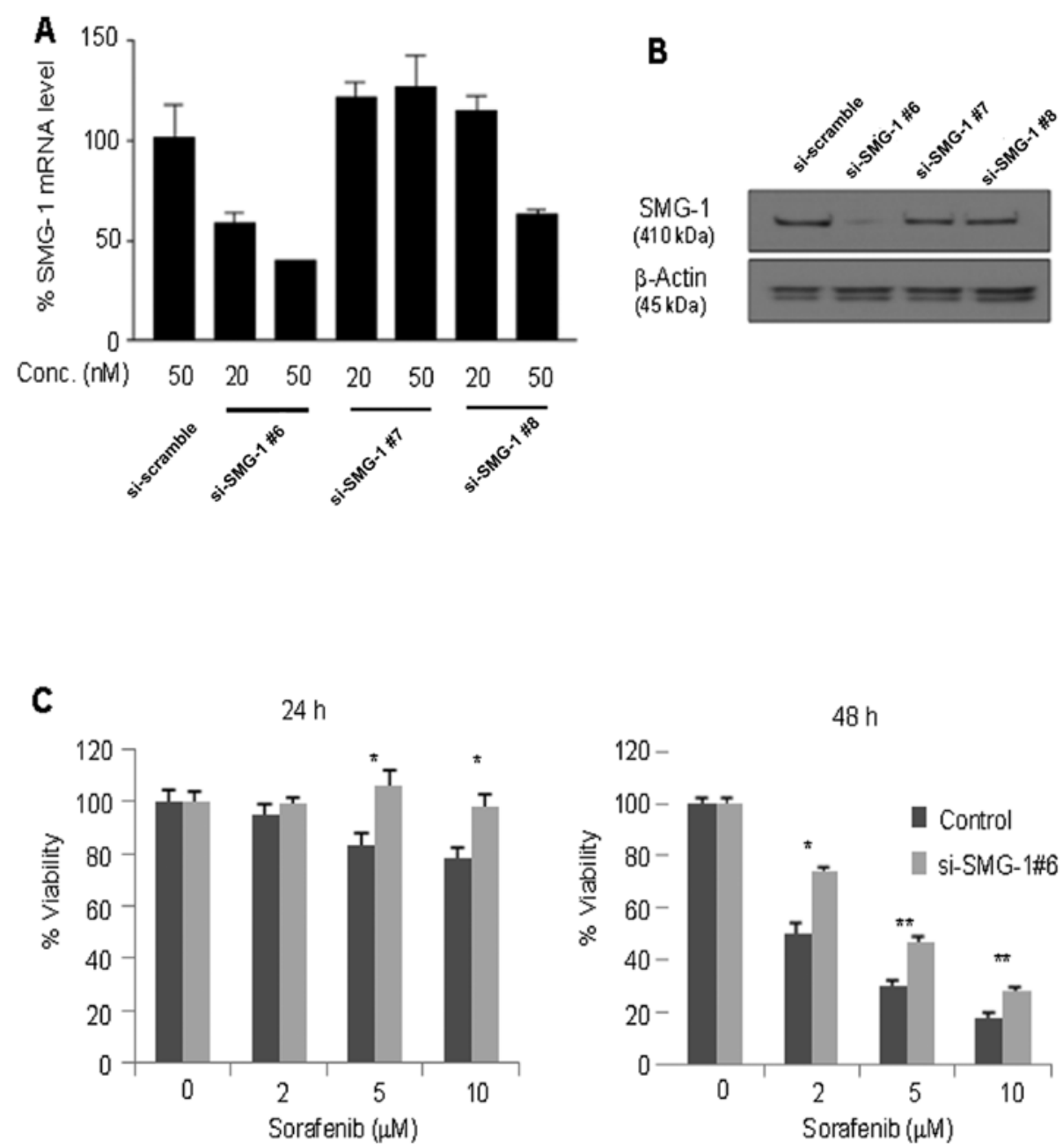

Figure 3. SMG-1 suppression induces sorafenib resistance in Hep3B cells. (A) The cells were transfected with $50 \mu \mathrm{M}$ of si-scramble, 20 or $50 \mu \mathrm{M}$ of three specific si-SMG-1 oligonucleotides named si-SMG-1 \#6, \#7 and \#8 for 2 days. Reverse transcription-PCR was measured by SMG-1 specific primers. (B) The cells were transfected with $50 \mu \mathrm{M}$ of si-scramble or si-SMG-1 \#6 for 2 days. Protein expression of SMG-1 was analyzed by anti-SMG-1 antibody. Primary antibody was diluted at 1:500 and secondary antibody was diluted at 1:2,000. (C) Cell viability was measured by EZ-cytox assays after 24 and $48 \mathrm{~h}$ in the presence of the indicated sorafenib treatments. ${ }^{*} \mathrm{p}<0.05 ;{ }^{* *} \mathrm{p}<0.01$ for comparison between the control and si-SMG-1 transfected cells treated with sorafenib at the indicated doses.

experiments using commercial siRNA of SMG-1 have revealed sorafenib resistance in siRNA-transfected hepatocellular cell lines compared to controls.

Hepatocarcinogenesis is a multistep process often initiated by external insults that lead to genetic aberrations in hepatocytes or stem cells, resulting in proliferation, apoptosis, dysplasia and neoplasia $(4,21,22)$. Significant progress in cancer molecular research technology has led to the development of various molecular approaches for the treatment of HCC. The identification of molecular targets involved in HCC pathogenesis and drug response continues to be an important area of active research.

Sorafenib is a potent antitumor agent that displays clinical activity against several solid tumors and is licensed for the treatment of patients with unresectable HCC. Its cytotoxic mode of action in HCC is mediated by inhibition of the RAS/MEK/ERK pathway, inhibition of tumor angiogenesis and induction of tumor cell apoptosis $(10,23)$. The effect of sorafenib on tumor size reduction was lower than anticipated, and it has shown only a minimal effect on survival and reduced disease progression in patients with advanced HCC
$(11,12,24)$. Hence, the importance of discovering genes related to resistance or non-responsiveness to sorafenib treatment in the hope of developing novel means of potentiating the drug's antitumor properties.

RNA interference (RNAi) is a naturally occurring mechanism that modulates gene expression at the post-transcriptional level and is a powerful tool to perform loss-of-function genetic screens for the identification of components of cellular signaling pathways (25-27). In eukaryotes, double-stranded interfering RNAs target complementary mRNAs for degradation, which results in the selective silencing of specific proteins. This characteristic of RNAi makes it an important laboratory work tool and has led to the characterization of novel oncogenes $(28,29)$. With the development of genome-wide RNA interference (RNAi) approaches, the cost and time that are involved in target identification, validation and other aspects of drug discovery have been significantly reduced.

SMG-1 is a serine-threonine kinase which plays a conserved role in nonsense-mediated mRNA decay (NMD) in worms and mammals, and human SMG-1 has also been implicated in the p53-mediated response to genotoxic stress 
A

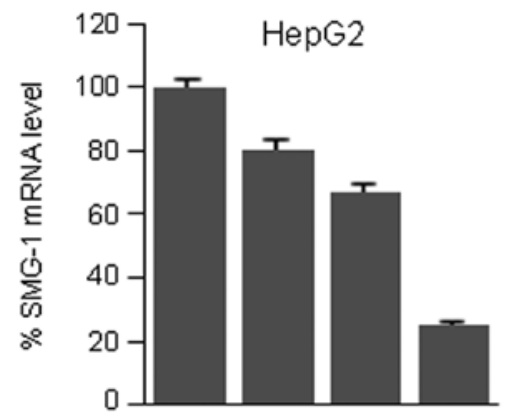

si-scramble (nM) 100 si-SMG-1 \#6 (nM)
20

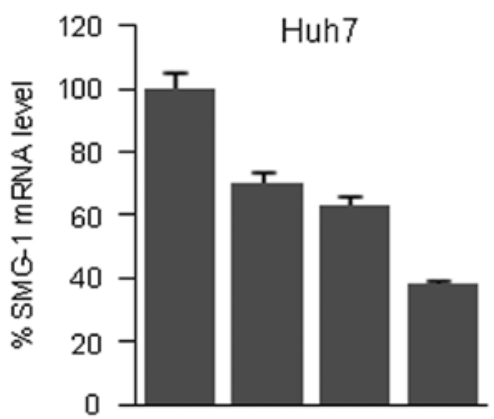

si-scramble (nM) 100

si-SMG-1 \#6 (nM)
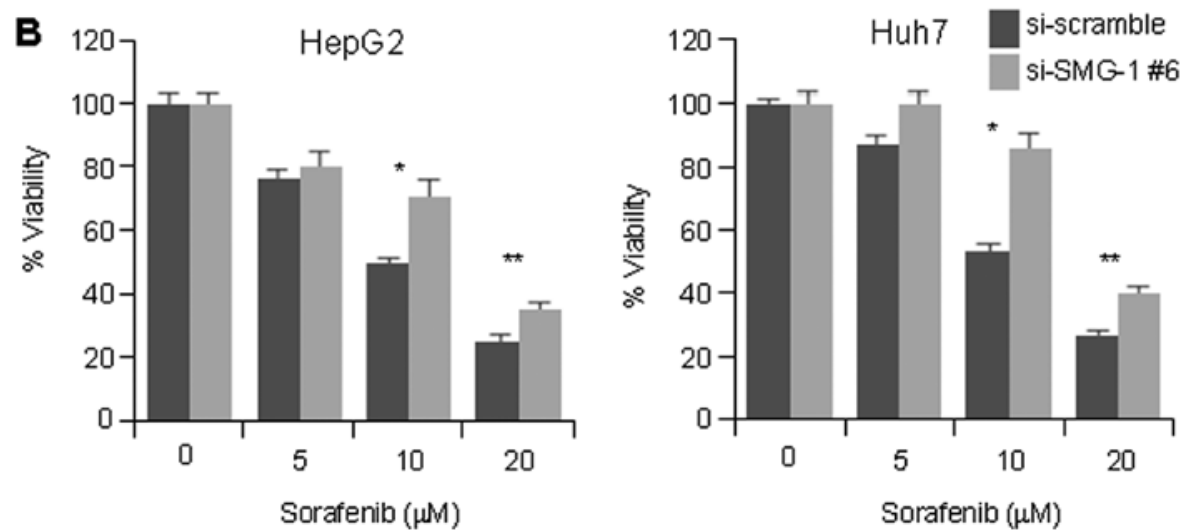

Figure 4. SMG-1 suppression induces sorafenib resistance in other HCC cell lines. (A) HepG2 and Huh7 cell lines were transfected with $100 \mu \mathrm{M}$ of si-scramble, 20 , 50 or $100 \mathrm{nM}$ of si-SMG-1 \#6 for 2 days. Reverse transcription-PCR were measured as described in Fig. 3. ${ }^{*} \mathrm{p}<0.05$; ${ }^{* * *} \mathrm{p}<0.01$ for comparison between the control and si-SMG-1 transfected cells treated with sorafenib at the indicated doses. (B) si-SMG-1 or negative control si-RNA transfected HepG2 cells (left panel) and Huh7 (right panel) were treated with sorafenib $(0,5,10$ and $20 \mu \mathrm{M})$ for $24 \mathrm{~h}$. Thereafter, cell viability was measured by EZ-cytox assay and compared. Mean \pm SD ${ }^{*} \mathrm{p}<0.05 ;{ }^{* *} \mathrm{p}<0.01$ for comparison between the control and si-SMG-1 transfected cells treated with sorafenib at the indicated doses.

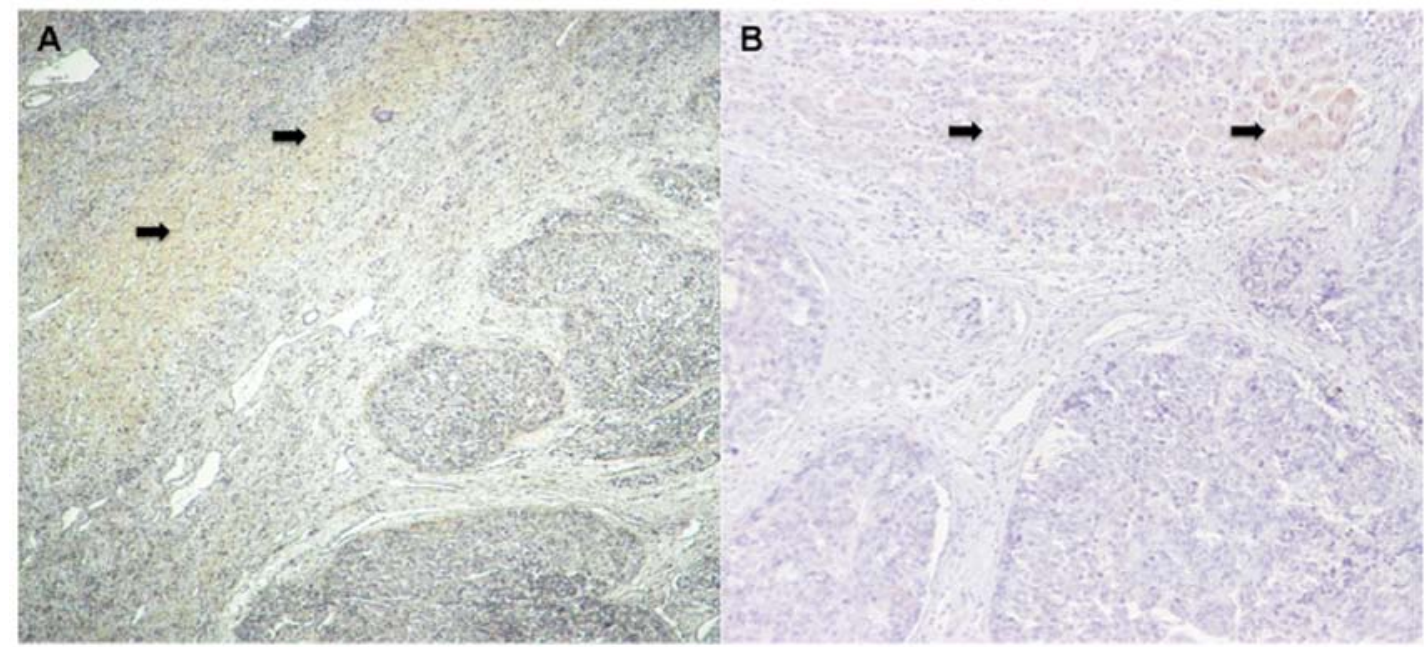

Figure 5. Immunohistochemical staining of resected cancer tissue. (A and B) Strong and diffuse cytoplasmic granular staining in non-cancerous cirrhotic hepatocytes (arrow) compared to cancer region. x100, magnification (representative data).

$(30,31)$. In addition, SMG-1 negatively regulates hypoxiainducible factor-1 (HIF-1) $(32,33)$ activity, which plays a central role in tumor progression by regulating genes involved in proliferation, glycolysis, angiogenesis and metastases, in hypoxia through MAPK activation (34). HIF-1 is one of the primary transcription factors responsible for increased 
gene expression in hypoxia, and vascular endothelial growth factor (VEGF), erythropoietin, and glycolytic enzymes are all targets of HIF-1 involved in the adaptive response of cells to hypoxia $(35,36)$. The hypervascularized nature of $\mathrm{HCC}$ implies a particular reliance on factors related to angiogenesis $(37,38)$. In several recent studies, VEGF was overexpressed in HCC, and HIF-1 played a central role in HCC progression and angiogenesis (39-41). Consequently, despite the complicated nature of tumor proliferation and the existence of multiple regulatory checkpoints, we hypothesize that knockdown of SMG-1 may potentiate angiogenesis or metastasis of HCC due to disinhibition of HIF-1.

Nenasheva et al (42) previously reported that defective SMG-1 kinase function might contribute to the process of malignant transformation in B cell lymphomas of different origins. Xia et al (43) recently reported that human SMG-1 was involved in gemcitabine-induced primary microRNA-155/BIC upregulation in a human pancreatic cancer cell line. Though little information about SMG-1 has been elucidated to date, further investigations of the precise action of SMG-1 in oncogenesis or chemotherapeutic responsiveness may be meaningful. In our present study, knockdown by RNAi of SMG-1 led to higher cell viability in sorafenib-treated Hep3B cells.

We performed RNAi knockdown on other HCC cell lines such as HepG2 and Huh7 cells, which resulted in a similarly increased cell viability. Although there were significant differences in cell viability between RNAi transfected and non-transfected cell lines, the survival rate of sorafenib-treated cancer cells was lower than anticipated. This may be due to the short reaction time and lower transfection efficiency of commercial RNAi itself, which is less of a consideration in the stable shRNA platform.

Immunohistochemical staining of SMG-1 in $\mathrm{HCC}$ tissues showed strong and diffuse cytoplasmic expression in non-cancerous cirrhotic region compared with cancerous region. These results suggest that SMG-1 expression is reduced during neoplastic transformation and also implies its role as a tumor suppressor gene. Loss of SMG-1 function may accelerate or convert malignant transformation potential to neoplasmic formation from uncertain causes. Therefore, we can postulate that SMG-1 modulates the response of HCC to sorafenib through effects on the RAS/MEK/ERK pathway, PI3K/Akt/mTOR pathway or HIF-1 $(34,44,45)$.

There are several limitations to consider in our experiment. Firstly, we could not determine anything about the underlying mechanisms or related signal transduction of SMG-1. Secondly, we could not show whether the potential regulation of SMG-1 by sorafenib is indirect or direct. Thirdly, we could not perform apoptotic analysis or other experiments to evaluate the role of SMG-1. Further molecular studies will be needed to investigate these properties.

In conclusion, this is the first report that utilizes the unbiased genetic approach of shRNA library screening to identify genes related to sorafenib resistance in HCC. These results show that using such a screening method is successful in identifying targets that have biological relevance to the pathogenesis and disease progressions of HCC. A potential regulator, SMG-1, was validated, and future studies can investigate its potential as an adjunctive clinical target to improve sorafenib therapy in patients with HCC.

\section{Acknowledgements}

This study was supported in part by grants from the Alberta Cancer Research Institute (23123), Canada Research Chair Program (95-203751), CIHR (MOP97962) and a fund (CMCDJ2011-P-007) of the Clinical Medical Research Institute from Daejeon St. Mary's Hospital, the Medical School of the Catholic University of Korea.

\section{References}

1. Parkin DM, Bray F, Ferlay $\mathrm{J}$ and Pisani P: Estimating the world cancer burden: Globocan 2000. Int J Cancer 94: 153-156, 2001.

2. El-Serag HB: Hepatocellular carcinoma. N Engl J Med 365: 1118-1127, 2011.

3. El-Serag HB and Rudolph KL: Hepatocellular carcinoma: epidemiology and molecular carcinogenesis. Gastroenterology 132: 2557-2576, 2007.

4. Farazi PA and DePinho RA: Hepatocellular carcinoma pathogenesis: from genes to environment. Nat Rev Cancer 6: 674-687, 2006.

5. Fattovich G, Stroffolini T, Zagni I and Donato F: Hepatocellular carcinoma in cirrhosis: incidence and risk factors. Gastroenterology 127: S35-S50, 2004.

6. Chen CJ, Yang HI and Iloeje UH: Hepatitis B virus DNA levels and outcomes in chronic hepatitis B. Hepatology 49: S72-S84, 2009.

7. Forner A, Reig ME, de Lope CR and Bruix J: Current strategy for staging and treatment: the BCLC update and future prospects. Semin Liver Dis 30: 61-74, 2010.

8. El-Serag HB, Marrero JA, Rudolph L and Reddy KR: Diagnosis and treatment of hepatocellular carcinoma. Gastroenterology 134: 1752-1763, 2008.

9. Wysocki PJ: Targeted therapy of hepatocellular cancer. Expert Opin Investig Drugs 19: 265-274, 2010.

10. Liu L, Cao Y,Chen C, et al: Sorafenib blocks the RAF/MEK/ERK pathway, inhibits tumor angiogenesis, and induces tumor cell apoptosis in hepatocellular carcinoma model PLC/PRF/5. Cancer Res 66: 11851-11858, 2006.

11. Llovet JM, Ricci S, Mazzaferro V, et al: Sorafenib in advanced hepatocellular carcinoma. N Engl J Med 359: 378-390, 2008.

12. Cheng AL, Kang YK, Chen Z, et al: Efficacy and safety of sorafenib in patients in the Asia-Pacific region with advanced hepatocellular carcinoma: a phase III randomised, doubleblind, placebo-controlled trial. Lancet Oncol 10: 25-34, 2009.

13. Trojniak MP, Palozzo AC, Mazurek M and Jirillo A: Sorafenib in hepatocellular carcinoma - a post marketing evaluation. Immunopharmacol Immunotoxicol 34: 419-422, 2012.

14. Lum L, Yao S, Mozer B, et al: Identification of Hedgehog pathway components by RNAi in Drosophila cultured cells. Science 299: 2039-2045, 2003.

15. Ashrafi K, Chang FY, Watts JL, et al: Genome-wide RNAi analysis of Caenorhabditis elegans fat regulatory genes. Nature 421: 268-272, 2003.

16. Iorns E, Lord CJ, Turner $\mathrm{N}$ and Ashworth A: Utilizing RNA interference to enhance cancer drug discovery. Nat Rev Drug Discov 6: 556-568, 2007.

17. Ahn BY, Elwi AN, Lee B, et al: Genetic screen identifies insulin-like growth factor binding protein 5 as a modulator of tamoxifen resistance in breast cancer. Cancer Res 70: 3013-3019, 2010.

18. Tang TC, Man S, Xu P, et al: Development of a resistance-like phenotype to sorafenib by human hepatocellular carcinoma cells is reversible and can be delayed by metronomic UFT chemotherapy. Neoplasia 12: 928-940, 2010.

19. Berns K, Hijmans EM, Mullenders J, et al: A large-scale RNAi screen in human cells identifies new components of the p53 pathway. Nature 428: 431-437, 2004.

20. Chen KF, Chen HL, Tai WT, et al: Activation of phosphatidylinositol 3-kinase/Akt signaling pathway mediates acquired resistance to sorafenib in hepatocellular carcinoma cells. J Pharmacol Exp Ther 337: 155-161, 2011.

21. Thorgeirsson SS and Grisham JW: Molecular pathogenesis of human hepatocellular carcinoma. Nat Genet 31: 339-346, 2002.

22. Villanueva A, Newell P, Chiang DY, Friedman SL and Llovet JM: Genomics and signaling pathways in hepatocellular carcinoma. Semin Liver Dis 27: 55-76, 2007. 
23. Wilhelm SM, Carter C, Tang L, et al: BAY 43-9006 exhibits broad spectrum oral antitumor activity and targets the $\mathrm{RAF} / \mathrm{MEK} / \mathrm{ERK}$ pathway and receptor tyrosine kinases involved in tumor progression and angiogenesis. Cancer Res 64: 7099-7109, 2004.

24. Kim JE, Ryoo BY, Ryu MH, et al: Sorafenib for hepatocellular carcinoma according to Child-Pugh class of liver function. Cancer Chemother Pharmacol 68: 1285-1290, 2011.

25. Fire A, Xu S, Montgomery MK, Kostas SA, Driver SE and Mello CC: Potent and specific genetic interference by doublestranded RNA in Caenorhabditis elegans. Nature 391: 806-811, 1998.

26. Chapman EJ and Carrington JC: Specialization and evolution of endogenous small RNA pathways. Nat Rev Genet 8: 884-896, 2007.

27. Kamath RS, Fraser AG, Dong Y, et al: Systematic functional analysis of the Caenorhabditis elegans genome using RNAi. Nature 421: 231-237, 2003.

28. Pan Q, Bao LW, Kleer CG, et al: Protein kinase C epsilon is a predictive biomarker of aggressive breast cancer and a validated target for RNA interference anticancer therapy. Cancer Res 65: 8366-8371, 2005.

29. Zhang Z, Jiang G, Yang F and Wang J: Knockdown of mutant K-ras expression by adenovirus-mediated siRNA inhibits the in vitro and in vivo growth of lung cancer cells. Cancer Biol Ther 5: 1481-1486, 2006.

30. Yamashita A, Ohnishi T, Kashima I, Taya Y and Ohno S: Human SMG-1, a novel phosphatidylinositol 3-kinase-related protein kinase, associates with components of the mRNA surveillance complex and is involved in the regulation of nonsense-mediated mRNA decay. Genes Dev 15: 2215-2228, 2001.

31. Masse I, Molin L, Mouchiroud L, et al: A novel role for the SMG-1 kinase in lifespan and oxidative stress resistance in Caenorhabditis elegans. PLoS One 3: e3354, 2008.

32. Keith B and Simon MC: Hypoxia-inducible factors, stem cells, and cancer. Cell 129: 465-472, 2007.

33. Kaelin WG Jr and Ratcliffe PJ: Oxygen sensing by metazoans: the central role of the HIF hydroxylase pathway. Mol Cell 30 393-402, 2008.
34. Chen RQ, Yang QK, Chen YL, et al: Kinome siRNA screen identifies SMG-1 as a negative regulator of hypoxia-inducible factor-1alpha in hypoxia. J Biol Chem 284: 16752-16758, 2009.

35. Wang GL, Jiang BH, Rue EA and Semenza GL: Hypoxia-inducible factor 1 is a basic-helix-loop-helix-PAS heterodimer regulated by cellular $\mathrm{O} 2$ tension. Proc Natl Acad Sci USA 92: 5510-5514, 1995.

36. Mottet D, Michel G, Renard P, Ninane N, Raes $M$ and Michiels C: ERK and calcium in activation of HIF-1. Ann NY Acad Sci 973: 448-453, 2002.

37. Pang R and Poon RT: Angiogenesis and antiangiogenic therapy in hepatocellular carcinoma. Cancer Lett 242: 151-167, 2006.

38. Kuboki S, Shimizu H, Mitsuhashi N, et al: Angiopoietin-2 levels in the hepatic vein as a useful predictor of tumor invasiveness and prognosis in human hepatocellular carcinoma. J Gastroenterol Hepatol 23: e157-e164, 2008.

39. Torimura T, Sata M, Ueno T, et al: Increased expression of vascular endothelial growth factor is associated with tumor progression in hepatocellular carcinoma. Hum Pathol 29: 986-991, 1998.

40. Nakamura K, Zen Y, Sato Y, et al: Vascular endothelial growth factor, its receptor Flk-1, and hypoxia inducible factor-1alpha are involved in malignant transformation in dysplastic nodules of the liver. Hum Pathol 38: 1532-1546, 2007.

41. Wu XZ, Xie GR and Chen D: Hypoxia and hepatocellular carcinoma: The therapeutic target for hepatocellular carcinoma. J Gastroenterol Hepatol 22: 1178-1182, 2007.

42. Nenasheva VV, Nikolaev AI, Martynenko AV, et al: Differential gene expression in HIV/SIV-associated and spontaneous lymphomas. Int J Med Sci 2: 122-128, 2005.

43. Xia QS, Ishigaki Y, Zhao X, et al: Human SMG-1 is involved in gemcitabine-induced primary microRNA-155/BIC up-regulation in human pancreatic cancer PANC-1 cells. Pancreas 40: 55-60, 2011.

44. Vivanco I and Sawyers CL: The phosphatidylinositol 3-Kinase AKT pathway in human cancer. Nat Rev Cancer 2: 489-501, 2002.

45. Fang JY and Richardson BC: The MAPK signalling pathways and colorectal cancer. Lancet Oncol 6: 322-327, 2005. 